\title{
pH-RESPONSIVE WOOD SLICES FOR THE CONTINUOUS SEPARATION OF LIGHT OIL/WATER/HEAVY OIL TRIPHASE MIXTURES
}

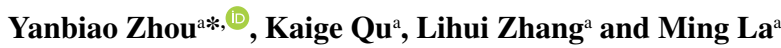 \\ aSchool of Chemistry and Environmental Engineering, Pingdingshan University, Pingdingshan, China
}

Recebido em 22/01/2021; aceito em 06/05/2021; publicado na web em 16/06/2021

\begin{abstract}
Light oil/water/heavy oil triphase mixtures are common in industrial wastewater, while nearly all separation materials with superwettability can only separate biphase mixtures of light oil/water or/and heavy oil/water. Here, a balsa wood slice with superhydrophilic/superoleophilic property in air was fabricated by a blade-cutting and ethanol-treating process. Therefore, the slice could be dually prewetted with both water and oil and thus showed underwater superoleophobic and underoil superhydrophobic properties without any further chemical modification. Such a slice showed underoil superhydrophobicity in nonbasic environment while transition into underoil superhydrophilicity under basic condition. Combining with the porous structure possessed by wood designed to transport liquids, this superwettability of the treated wood slice could be leveraged in the continuous separation of light oil/water/heavy oil triphase mixtures only driven by gravity with a high permeation flux and separation efficiency. After each separation, the slice was easily recovered by washing with acidic solution, and it could be recycled up to 15 times without any loss of $\mathrm{pH}$ responsibility. During repeated cycling separation, the slice exhibited excellent separation stability.
\end{abstract}

Keywords: wood; pH-responsive; triphase mixtures; continuous separation.

\section{INTRODUCTION}

Large amounts of oily wastewater generated by industrial production and daily life have caused both resource waste and environmental pollution. ${ }^{1,2} \mathrm{An}$ increasing demand for the treatment of oily wastewater is driving the need for oil/water separation process. Over recent years, porous materials with superwettability have been extensively studied for oil/water separation. ${ }^{3,4}$ Much attention has been given to biphase separation materials, which were applied to separate light oil/water or/and heavy oil/water mixtures., In general, superhydrophobic and underoil superhydrophobic membranes are suitable for separating heavy oil/water mixtures, while underwater superoleophobic meshes are suited to separate light oil/water mixtures..$^{7-9}$ Most of the previous investigations have been conducted on the separation of individual oil type-based mixtures, but studies of separating materials that can be used to continuously separate triphase mixtures are largely limited. ${ }^{10}$ However, much of oily sewage is light oil/water/heavy oil triphase mixtures. To address this issue, Ju group prepared a $\mathrm{pH}$-dependent foam for continuously separating oil/water/oil triphase mixtures through surface modification with mixed thiols of $\mathrm{HS}\left(\mathrm{CH}_{2}\right)_{9} \mathrm{CH}_{3}$ and $\mathrm{HS}\left(\mathrm{CH}_{2}\right)_{10} \mathrm{COOH} .{ }^{11}$ Similarly, Dang and coworkers also designed a smart membrane by dip-coating a copolymer containing $\mathrm{pH}$-responsive block to continuously separate of oil/water/oil triphase mixtures. ${ }^{12}$ Feng et al. reported the fabrication of a smart $\mathrm{pH}$ responsive nylon membrane with $\mathrm{pH}$-responsibility by selfassembling thiol molecules for the separation of light oil/water/ heavy oil mixture. ${ }^{13}$ These material surfaces were modified by introduction of ionizable groups of carboxylic acids, which could reversibly switch wettability using $\mathrm{pH}$ as the trigger. However, their coating films easily peel off from their surface during separation and result in a partial loss of response to $\mathrm{pH}$ stimulus, which impedes their reversible application in separation equipment. ${ }^{14}$ Hence, it is highly desirable to find novel triphase separation materials without surface modification to solve the problem.
Most woods possess an oriented porous structure constituted by aligned tubular cells mainly consisting of amphiphilic cellulose, lignin and hemicellulose. The natural amphiphilic components and tubular structure enable them as a water-removing and oil-removing material for successful separation of light oil/water and heavy oil/ water mixtures. ${ }^{15,16}$ Besides, the $\mathrm{pH}$ of most woods is slightly acidic, such as pinus -4.6 , sweetgum -5.3 and paulownia - 6.0. Among them, balsa wood exhibits a weak acidic character with $\mathrm{pH}$ value at 5.5. This behavior was caused by acidic type functional groups, especially carboxylic acids. ${ }^{17}$ The tubular structure along with the acidic character positions balsa wood as a promising candidate for separating triphase mixtures.

In this respect, our purpose is to use woods that have different kinds of acidic groups distributed in the cell wall to continuously separate triphase mixtures. Here, we chose balsa wood as separation material because of its porous structure, amphiphilic composition and rich acidic groups. ${ }^{15}$ Here, a wood slice with superhydrophilic/ superoleophilic property was successfully fabricated by a bladecutting and solvent-treating process. Firstly, the basswood was sawed into slices, two sides of which were trimmed with a sharp blade. Secondly, the natural basswood was immersed in ethanol and dried under room temperature. The resulting slice showed underwater superoleophobicity/underoil superhydrophobicity. When the $\mathrm{pH}$ of water was changed from acidic to basic, the slice underwent an underoil superhydrophobicity-to-underoil superhydrophilicity transition. The slice had good performance for continuous separation of light oil/water/heavy oil triphase mixtures, and it was a promising wood material for high-efficient oil/water separation (Scheme 1).

\section{MATERIAL AND METHODS}

\section{Materials}

Fresh balsa lumber was purchased from a local wood products manufacturer. Ethanol, n-hexane, toluene, chloroform, dichloromethane, 1,2-dichloroethane, hydrochloric acid and sodium 


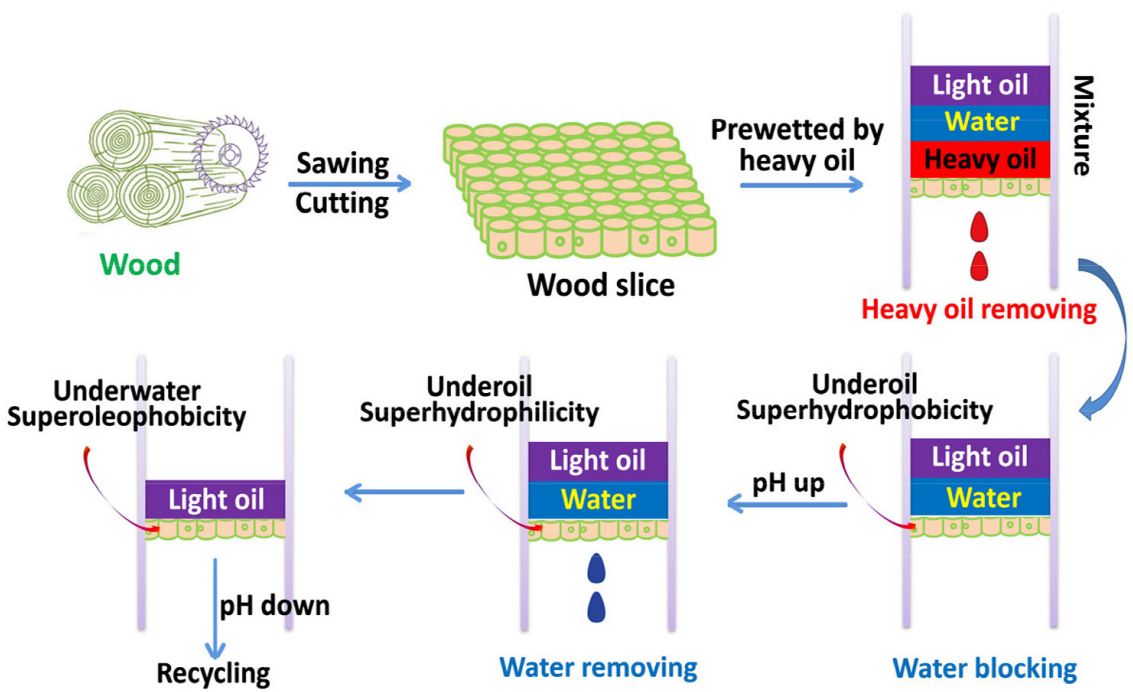

Scheme 1. Preparation of the wood slice for the separation of light/water/heavy oil/water triphase mixtures

hydroxide were purchased from purchased from Tianjin Chemical Reagent Factory. Kerosene was used as received from Sinopec Group.

\section{Preparation of wood slice}

Balsa wood was sawed into slices from a fresh log along the transverse direction by circular saw. To clear residual sawdust and debris, the two sides of the sawed slice were trimmed with a sharp razor blade. The slice was then soaked in $200 \mathrm{~mL}$ ethanol for $0.5 \mathrm{~h}$ to remove soluble components and was taken out and dried in an air oven at $50{ }^{\circ} \mathrm{C}$ for $0.5 \mathrm{~h}$ before use. The cooperative effect of massive amphiphilic groups and hierarchical surface structure endowed the natural wood with underwater superoleophobicity and underoil hydrophobicity. After treatment, the underoil hydrophobicity of the slice was converted to underoil superhydrophobicity. Because wood had a weak acidic character, the slice showed underoil superhydrophobicity to acidic and neutral water and underoil superhydrophilicity to basic water. This superwettability, combined with its natural permeability, could be exploited to continuously separate light oil/water/heavy oil triphase mixtures.

\section{Separation of triphase mixtures}

The treated wood slice was fixed between two glass tubes with the same diameter, above which, a dropping funnel with a valve was fixed. Three types of light oil/water/heavy oil triphase mixtures, including kerosene/water/chloroform, toluene/water/ dichloromethane and hexane/water/dichloroethane, were separated by this device. The oils and water were dyed with oil red and methylene blue, respectively. A $120 \mathrm{~mL}$ of light oil/water/heavy oil triphase mixtures $(1: 1: 1, \mathrm{~V}: \mathrm{V}: \mathrm{V})$ was added to the dropping funnel. After prewetting the slice with heavy oil, open the valve of the dropping funnel to allow the triphase mixtures to flow into the upper tube. The slice initially came into contact with the heavy oil, which passed through the slice and was collected in a designated beaker through the below tube. No water was observed entering into the beaker through the slice. The $\mathrm{pH}$ of the water was then shifted from acidic or neutral to basic by the addition of $\mathrm{NaOH}$ solution. After basification for about 15 seconds, the water layer passed though the slice and was collected in a designated beaker. Finally, only upmost light oil was repelled and collected in the upper glass tube. In this way, light oil, water and heavy oil mixture were separated. As a very important parameter for oil/water mixture separation, the flux of oils and water permeating through the slice was calculated by the following formula (1):

$$
F=\frac{V}{A \times t} \times 100 \%
$$

where $F$ represents the permeation flux during the filtration process $\left(\mathrm{L} \mathrm{m}^{-2} \mathrm{~h}^{-1}\right), V$ is the volume of permeated liquids $(\mathrm{L}), A$ is the valid area of the slice $\left(\mathrm{m}^{2}\right)$ and $t$ is the required time (h) for separating mixtures while keeping the height of the feed solution constantly at $10 \mathrm{~cm}$. Then the oil/water separation efficiency was calculated in terms of the liquid rejection coefficient $(R)$ from the following equation (2):

$$
R=\left(1-\frac{C_{\mathrm{f}}}{C_{\mathrm{o}}}\right) \times 100 \%
$$

where $C_{\mathrm{f}}$ and $C_{\mathrm{o}}$ denote the oil (water) concentration in the water (oil) samples after separation and the equivalent oil concentration of the mixture before separation, respectively.

\section{Characterizations}

The structures of the balsa slices were observed by a field emission scanning electron microscopy (FESEM, Hitachi SU8000). The contact angle measurement (PD-200, Attension Theta System, Sweden) was adopted to measure the slice surface wettability at room temperature. The contact angle of droplets $(3 \mu \mathrm{L}$, measured with a microsyringe) on three different regions was measured $10 \mathrm{~s}$ after the contact angle becoming virtually constant on the slice surface, and the average angles were calculated to determine the wettability of the slice. The surface chemical composition was investigated by a Brucker Tensor 27 FTIR spectrometer (Bruker Optics, Germany). Photographs of the separation process were taken with a digital camera (D7000, Nikon). The water content in collected oils was accurately analyzed using Karl Fischer Titrator (YT-200, China). The oil content in the collected water was determined by infrared oil content analyzer (Oil 460, China).

\section{RESULTS AND DISCUSSION}

After sawing and cutting, the dried pristine balsa wood transverse sections obtained are not covered by folded and shredded cell wall fragments (Figure 1a). Open cells naturally have a hierarchical 
structure, which is composed of well-oriented nanoscale fibrils (3-5 nm wide) and microscale cell walls $\left(2-4 \mu \mathrm{m}\right.$ wide). ${ }^{18}$ This hierarchical structure is generally required for obtaining superwettability. The average cell length, diameter and wall thickness of the wood are around $0.65 \mathrm{~mm}, 30 \mu \mathrm{m}$ and $3 \mu \mathrm{m}$, respectively. Because the cell length of around $0.65 \mathrm{~mm}$ was shorter than the thickness of the slice $(1 \mathrm{~mm}),{ }^{19}$ no hollow tubular cell would be cut open from both ends. Although some bordered pits on the longitudinal section were blocked, fluid still can flow from one cell to the next through open circular bordered pits (about $4 \mathrm{um}$ ) following a zigzag path during separation (Figure 1b). ${ }^{20}$ After treatment with ethanol, it can be seen that more lamella were released due to the extraction of soluble waxes between them by ethanol. That was to say, extraction of waxes caused crack in the cell wall section and seemed to facilitate the separation of the cell wall into lamella (Figure 1c). ${ }^{21}$ This increased section roughness caused by the treatment is more beneficial for obtaining superwettable surface. For the the longitudinal section, the treated slice had a similar texture with that of the pristine slice (Figure 1d).

The functional groups present in the natural wood slice were investigated by the FTIR spectra (Figure 2a). The strong peak at $3329 \mathrm{~cm}^{-1}$ was originated from the stretching vibration of O-H group. The sharp peaks at about 2943 and $2894 \mathrm{~cm}^{-1}$ were ascribed to the stretching vibration of C-H group. The peaks at 1601, 1504, 1456 and $1260 \mathrm{~cm}^{-1}$ corresponded to the stretching vibration of aromatic and $\mathrm{C}-\mathrm{H}$ groups. ${ }^{22}$ The peaks at 1155,1107 and $1029 \mathrm{~cm}^{-1}$ were assigned to $\mathrm{C}-\mathrm{O}$ group. The peak at $1739 \mathrm{~cm}^{-1}$ belonged to the stretching vibration of $\mathrm{C}=\mathrm{O}$ groups include mainly ester group and carboxylic group. ${ }^{23}$ The carboxylic group is a typical $\mathrm{pH}$-responsive functional group, which can accept or donate protons upon the $\mathrm{pH}$ change. These peaks implied that the wood had a mass of hydrophilic $(-\mathrm{OH}$, $\mathrm{C}-\mathrm{O}, \mathrm{C}=\mathrm{O})$, oleophilic $(\mathrm{C}-\mathrm{H})$ and $\mathrm{pH}$-responsive $(-\mathrm{COOH})$ group. ${ }^{24}$ Combining with micro/nanoscale hierarchical rough section, these hydrophilic and oleophilic functional groups endowed the wood with underoil superhydrophobicity and underwater superoleophobicity.
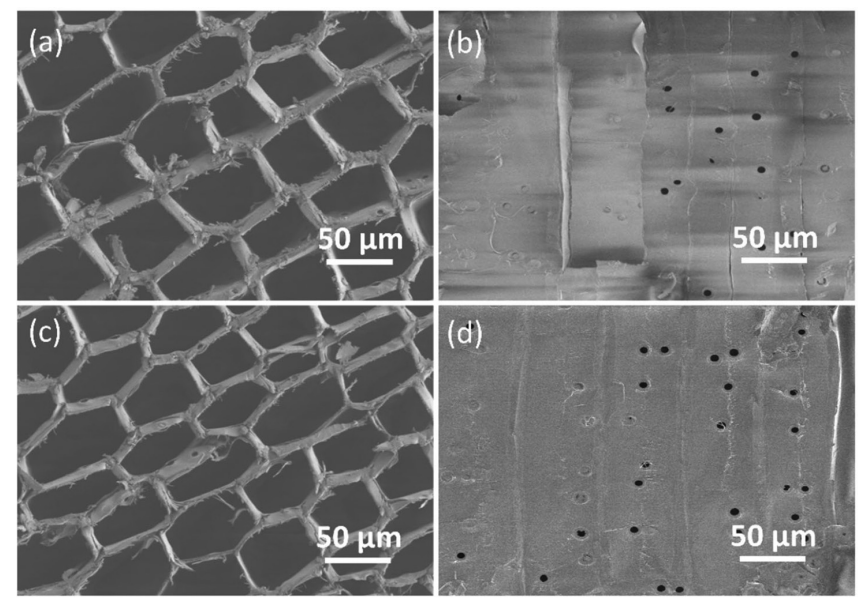

Figure 1. FESEM images of balsa slices. (a) transverse section and (c) longitudinal section of the pristine slice, (c) transverse section and $(d)$ longitudinal section of the treated slice

After treatment by ethanol, the slice had a similar FTIR spectrum with that of the pristine slice (Figure 2a).

The surface wettability of any solid including porous materials is usually quantified by measuring the contact angle of a droplet deposited on the solid surface. The surface wetting behavior of the balsa wood slice before and after treatment was evaluated by contact angle measurement. After water and oil droplets were directly dropped onto the pristine and treated slice surface under oil (kerosene) and under water, the response of the slice surface wettability to $\mathrm{pH}$ changes and their different stable time on the slice was investigated. When an acidic or neutral water droplet was dropped onto the pristine slice surface in oil, the water droplet appeared to be spheroid shape, indicating that the slice had underoil hydrophobicity (Figure 2b). When an alkaline water droplet was dropped to the slice surface, the water droplets spread out on the
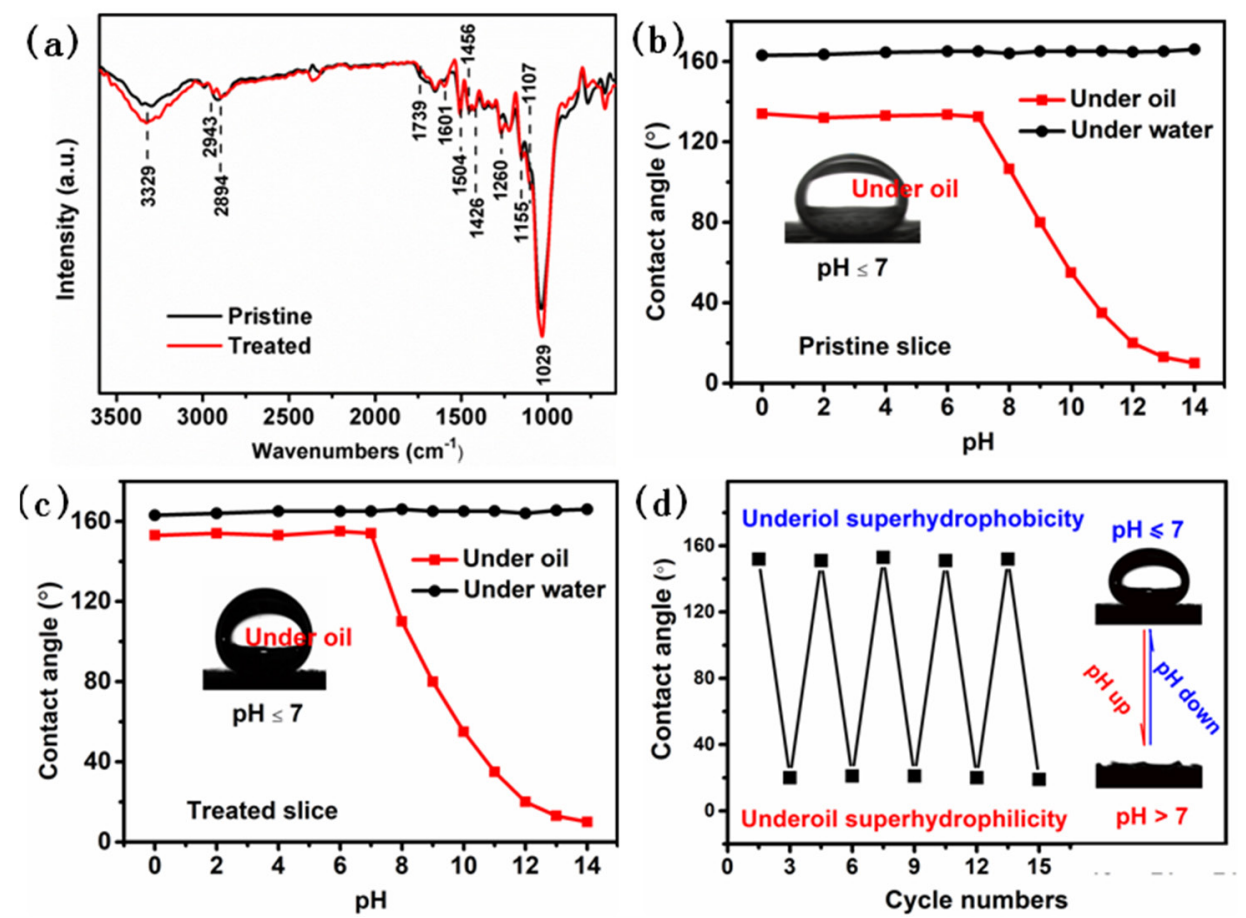

Figure 2. The pH-responsive wettability of the balsa wood slice. (a) The FTIR spectra of the slice before and after treatment. (b, $c$ )The variations of underoil (kerosene) water contact angles with water pH for the pristine and treated slice. (d) Reversible water wettability of the treated slice switched between underoil superhydrophobicity and underoil superhydrophilicity by altering water $\mathrm{pH}$ 
slice surface. In comparison, the acidic or neutral water droplet maintained sphere shape on the treated slice surface with water contact angle higher than $150^{\circ}$ for more than $200 \mathrm{~s}$, indicating the slice had good underoil superhydrophobicity (Figure 2c). The basic water droplet was completely absorbed into the slice within different time. This phenomenon might be attributed to the deprotonation process of acidic type functional groups in the slice, such as the carboxylic acid groups. For example, the basic water droplet at high $\mathrm{pH}=12$ was completely absorbed into the treated slice within tens of seconds, indicating its wettability quickly changed to underoil superhydrophilicity. However, as an oil droplet was directly placed on the surface of the slice under water at different $\mathrm{pH}$ values, the droplet held spherical shape with water contact angles larger than $160^{\circ}$ for periods longer than 200 s. It was noted that when the alkalized slice was washed with $\mathrm{HCl}$ solution and dried, the wettability was recovered from underoil superhydrophilicity to underoil superhydrophobicity with a water contact angle of above $150^{\circ}$. Based on the $\mathrm{pH}$-responsive behavior, the wettability of the slice could be alternated between underoil superhydrophobicity and underoil superhydrophilicity without loss in the $\mathrm{pH}$-responsive performance by rinsing the basic water-wetting slice with acidic water and drying in an oven at $50{ }^{\circ} \mathrm{C}$ (Figure $2 \mathrm{~d}$ ). The treated slice still remained its excellent $\mathrm{pH}$ responsibility after acid and base treatment after 15 cycles. The changeable wettability might be applied in successive separation of light oil/water/heavy oil triphase systems.

The wettability of the treated slice enabled selective collection of water and oils from triphase mixtures of light oil/water $(\mathrm{pH} \leq 7) /$ heavy oil. The slice was prewetted with heavy oil to form an oilwetting slice, which exhibited underoil superhydrophobicity. When separating the light oil/water/heavy oil triphase mixtures, the slice first came into contact with heavy oil (Figure 3a), which permeated through the oil-wetting slice but water was blocked, thus allowing the heavy oil to pass into the designated beaker (Figure $3 \mathrm{~b}$ ). The $\mathrm{pH}$ of the water was then shifted from acidic or neutral to basic by the addition of basic solution (Figure 3c). This alkaline treatment changed the wettability of the slice from underoil superhydrophobicity to underoil superhydrophilicity, resulting in rapid permeation of the water through the slice (Figure 3c). Finally, after all the water was collected, the upper light oil was collected in the upper glass tube (Figure 3d). The effectiveness of the separation based on the treated slice can be attributed to the regularly distributed cells and their chemical composition. The combination of tubular cells and amphiphilic composition produced superwetting phenomena, trapping water and oil when submerged in the two liquids without the formation of a trapped air layer. This effect resulted in either a water or oil layer becoming trapped within the slice. Besides, there are different kinds of acidic compounds such as acidic carbohydrates distributed in the cell wall. These compounds form more hydrophilic salts with base when exposed to alkaline water, resulting in the wettability change from underoil superhydrophobicity to underoil superhydrophilicity. The $\mathrm{pH}$-responsive wettability of the slice successfully realized the sequential separation and collection of individual components from triphase mixture.

Considering the variable composition of oils, a variety of light oil/water/heavy oil mixtures, including kerosene/water/chloroform, toluene/water/dichloromethane and hexane/water/dichloroethane mixtures, were separated successfully with the treated slice. Figure 4a shows the flux of the treated slice for three types of light oil/water/heavy oil mixtures. The flux of heavy oils (chloroform, dichloromethane, dichloroethane) was higher than that of light oils (kerosene, toluene, hexane). Taking a kerosene/water/chloroform mixture as an example, the separation flux at different separation cycles are provided in Figure $4 \mathrm{~b}$. The separation flux of water slightly decreased from 3400 to $3150 \mathrm{~L} \mathrm{~m}^{-2} \mathrm{~h}^{-1}$ after being recycled 15 times due to oil pollution of the slice. This indicated that the separation flux of the slice could be $93 \%$ recovered by simply washing with ethanol and drying. Therefore, it can be easily reused. As displayed in Figure $4 \mathrm{c}$, the separation efficiency of all three oil/water mixtures was above $99.5 \%$ for both water and oil removal, thus exhibiting excellent separation properties. Taking the kerosene/water/chloroform mixture for example, the effect of separation cycle on the separation efficiency of the slice is shown in Figure 4d. The separation efficiency remained fairly high $(>99.5 \%)$ in 15 cycles for the mixture, which possessed good light oil/water/heavy oil separation reusability.

\section{CONCLUSIONS}

In conclusion, a smart wood slice with tunable wettability had been fabricated by a facile blade-cutting and ethanol-treating method. The treated slice allowed the wettability to be switched between underwater superoleophobic and underoil superhydrophilic properties just by adjusting the $\mathrm{pH}$ of the aqueous phase. The slice that integrated the $\mathrm{pH}$-regulated surface wettability and tubular structures of the slice realized continuous separation for light oil/water/heavy oil mixtures. The synergistic effect can separate multiple triphase mixtures with high permeation flux and separation efficiency. Moreover, the structure and components rendered the slice with recyclability for
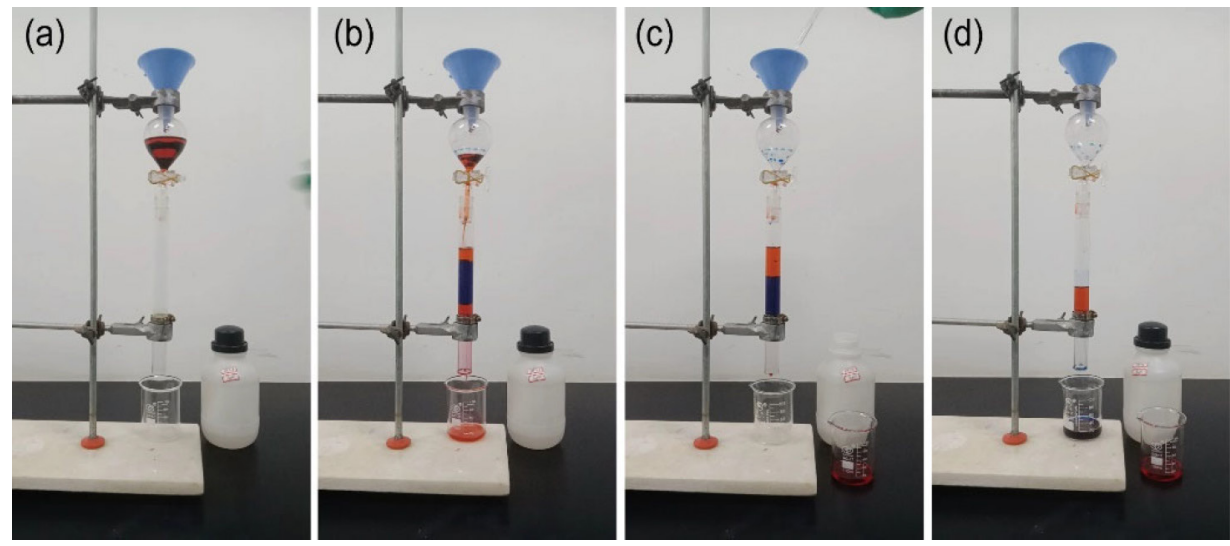

Figure 3. Photographs of continuous separation process of a light oil/water/heavy oil triphase mixture. (a) Prewetting the slice with heavy oil, a triphase mixture of kerosene/acidic or neutral water/chloroform flowed into the upper tube through a dropping funnel. (b) Chloroform permeated the slice into a beaker while the acidic or neutral water was rejected on the slice. (c) After regulating the $\mathrm{pH}$ value from acidic or neutral to basic, the basic water layer passed through the slice. (d) The upper oil layer was retained in the upper tube. Two oil layers were colored by oil red and water layer was dyed with methylene blue 
(a)

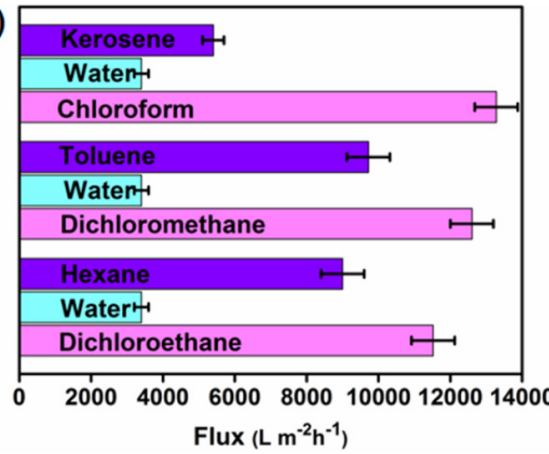

(c)

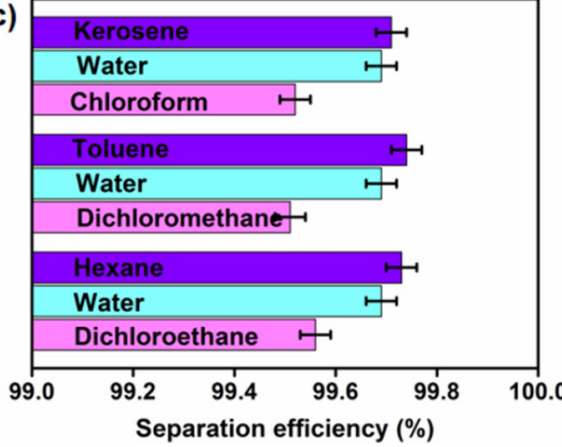

(b)
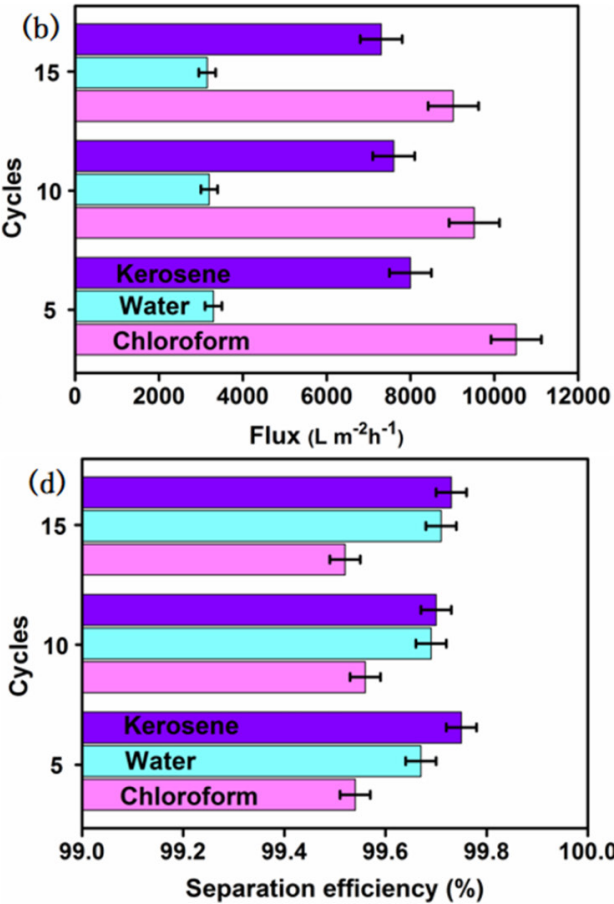

Figure 4. Permeation flux and separation efficiency of gravity-driven separation process using the treated slice upon the pH variation. (a) Flux of the slice for three types of triphase mixtures. (b) Flux of the slice for 15 kerosene/water/chloroform mixture separation cycles. (c) Separation efficiency of the slice for three types of oil/water/heavy oil mixtures. (d) Separation efficiency of the slice for 15 kerosene/water/chloroform mixture separation cycles

the long-term separation. Similar tubular structures and weak acidity possessed by most woods might possibly make them a promising candidate for separating triphase mixtures.

\section{SUPPLEMENTARY MATERIAL}

A video of the continuous separation process of a light oil/water/ heavy oil triphase mixture is available at http://quimicanova.sbq.org.br, in .mp4 format, with free access.

\section{ACKNOWLEDGEMENTS}

The Natural Science Foundation of Henan Province of China (162300410208), the Foundation of Henan Educational Committee (16A150058), the High-level Talent Foundation of Pingdingshan University (PXY-BSQD-2015003) and the Young Backbones of the College Teachers in Henan Province (2020GGJS227).

\section{REFERENCES}

1. Gupta R. K.; Dunderdale G. J.; England M. W.; Hozumi, A.; J. Mater. Chem. A 2017, 5, 16025.

2. Yong J.; Huo J.; Chen F.; Yang Q.; Hou X.; Phys. Chem. Chem. Phys. 2018, 20, 25140.

3. Fu, Q.; Ansari, F.; Zhou, Q.; Berglund, L. A.; ACS Nano 2018, 12, 2222.

4. Deng, Y.; Peng, C.; Dai, M.; Lin, D.; Ali, I.; Alhewairini, S. S.; Zheng, X.; Chen, G.; Li, J.; Naz, I.; J. Clean. Prod. 2020, 266, 121624.

5. Bian H.; Yong J.; Yang Q.; Hou X.; Chen F.; Front. Chem. 2020, 8, 507.

6. Qiu L.; Sun Y.; Guo Z.; J. Mater. Chem. A 2020, 8, 16831.
7. Ma, T.; Li, L.; Mei, C.; Wang, Q.; Guo, C.; J. Mater. Sci. 2021, 56, 5624.

8. Chen C.; Weng D.; Mahmood A.; Chen S.; Wang J.; ACS Appl. Mater. Interfaces 2019, 11, 11006.

9. Li J.; Xu C.; Guo C.; Tian H.; Zha, F.; Guo L.; J. Mater. Chem. A 2018, $6,223$.

10. Cao, G.; Zhang, W.; Jia, Z.; Liu, F.; Yang, H.; Yu, Q.; Wang, Y.; Di, X.; Wang, C.; Ho, S. H.; ACS Appl. Mater. Interfaces 2017, 9, 36368.

11. Ju G.; Cheng M.; Shi F.; NPG Asia Mater. 2014, 6, e111.

12. Dang Z.; Liu L.; Li Y.; Xiang Y.; Guo G.; ACS Appl. Mater. Interfaces 2016, 8,31281

13. Li, X.; Zhang, Q.; Zhang, W.; Qu, R.; Wei, Y.; Feng, L.; Adv. Mater. Interfaces 2018, 5, 1801179.

14. El-Samak, A. A.; Ponnamma, D.; Hassan, M. K.; Ammar, A.; Adham, S.; Al-Maadeed, M. A. A.; Karim, A.; Polym. Rev. 2020, 60, 671.

15. Zhou, Y. B.; Qu, K. G.; Zhang, L.; Luo, X. Q.; Liao B. H.; J. Dispersion Sci. Technol. 2019, 37, 1532.

16. Jiang, F.; Hsieh, Y. L.; J. Mater. Chem. A 2014, 2, 6337.

17. Shoulaifar T. K.; DeMartini N.; Ivaska A.; Fardim P.; Hupa M.; Bioresour. Technol. 2012, 123, 338.

18. Luo, B.; Yang, S.; Zhao, G.; J. Southwest Forest. Univ. 2011, 31, 59.

19. Borrega M.; Ahvenainen P.; Serimaa R.; Gibson L.; Wood Sci. Technol. 2015, 49, 403.

20. Yang X.; Berglund L. A.; Adv. Mater. 2020, 2001118.

21. Rana, M.; Chen, J. T.; Yang, S.; Ma, P. C.; Adv. Mater. Interfaces 2016, 3,1600128

22. Fahey L. M.; Nieuwoudt M. K.; Harris P. J.; Cellulose 2017, 24, 5275.

23. Owen, N. L.; Thomas, D. W.; Appl. Spectrosc. 1989, 43, 451.

24. Campbell W. G.; Bryant S. A.; Nature 1941, 147, 357. 